\title{
Significance of microalgal-bacterial interactions for aquaculture.
}

\begin{abstract}
Knowledge on the importance of associations between bacteria and microalgae in aquatic ecosystems is rather limited at the moment, mostly due to a lack of studies at the molecular and biochemical level of microorganisms. This paper discusses the current knowledge on microalgae-bacteria interactions and their potential impacts on the productivity, efficiency and sustainability of aquaculture. Current findings suggest that the interactions are complex and specific. The release of stimulatory products by bacteria that enhance the growth of microalgae, and vice versa, indicates the existence of mutualistic relations. Other factors, such as signalling between bacteria and microalgae, may also play an important role. Although these interactions may be of significant importance, to date, only a few findings have been reported on the use of consortia consisting of microalgae and bacteria for practical purposes. Interestingly, these results pointed out that a combination of microalgae and bacteria is often better than using either of them alone. Further research is needed to obtain a thorough understanding of the mechanisms behind the interactions between these microorganisms, including the identification of active compounds. This knowledge will enable the selection of appropriate consortia for different applications in aquaculture, including disease control and high and sustainable production of feed.
\end{abstract}

Keyword: Aquaculture; Bacteria; Consortium; Greenwater; Microalgae; Mutualism; Quorum sensing interference. 